\title{
Characterization and Evaluation of Activation Energy for Dc Conductivity of Polypyrrole/Nickel Zinc Iron Oxide Nanocomposites
}

\author{
*V.S. Shanthala ${ }^{1}$, S.N. Shobha Devi ${ }^{2}$, M. V. Murugendrappa ${ }^{3}$, \\ H. Nagabhushana ${ }^{4}$ \\ ${ }^{I}$ The Oxford College of Science, Department of Physics, Bangalore, Karnataka, India \\ ${ }^{2}$ Department of Physics, BMS College for Women, Bangalore, Karnataka, India \\ ${ }^{3}$ Department of Physics, BMS College of Engineering, Bangalore, Karnataka, India \\ ${ }^{4}$ Department of Studies and Research in Physics, Tumkur University, Tumkur, Karnataka, India \\ Corresponding Author: V.S. Shanthala
}

\begin{abstract}
Polymer nanocomposites of Polypyrrole and Nickel zinc iron oxide $\left(\mathrm{NiZnFe}_{2} \mathrm{O}_{4}\right)$ are prepared by insitu polymerisation using Ammonium persulphate as oxidising agent. Various nanocomposites have been prepared by varying the level of additive material $\mathrm{NiZnFe}_{2} \mathrm{O}_{4}$. The formation of nanocomposites and changes in the structural and microstructural properties were investigated by using XRD, FTIR, and SEM analysis. The size of the particle was analysed by XRD using Scherrer equation and found to be in the range of $15 \mathrm{~nm}-20 \mathrm{~nm}$. The lattice parameters were evaluated by W-H plots. DC conductivity has been measured in which activation energy (Ea) of the composites has been evaluated. Activation energies were evaluated from Arrhenius plots for all compositions. Results show that the incorporation of additive material increases the activation energy ' $E a$ ' for the DC conductivity of the composites, compared to the base polymer. The increase in the activation energy ' $E a$ ' is found to depend on the level of the additive in the composites. Significant increase (196\%) in DC conductivity was observed for nanocomposite of $50 \mathrm{wt} . \%$. Therefore such polymer nanocomposites find applications in microwave absorbers, sensors, electromagnetishielding etc.,
\end{abstract}

Keywords: Polypyrrole, nanocomposite, conductivity, $\mathrm{NiZnFe}_{2} \mathrm{O}_{4}$, Activation energy, XRD.

\section{Introduction}

In recent years Polymer nanocomposites has been a field of intense study, all over the world due to its unusual properties. Owing to their unusual combination of properties they have wide range of applications in various fields such as sensors, electronic devices, memory devices, lithium batteries, Energy storage, Microwave absorbers, electromagnetic shielding, etc. Besides their improved properties, these nanocomposites materials are also easily molded to near-final shape, simplifying their manufacturing [1-2]. Conducting polymers are the class of polymer which possess good tuneable electrical conductivity. But they are chemically sensitive and have poor mechanical properties; on the other hand, nanomaterials possess good mechanical properties. Thus nanocomposites formed by reinforcement of conducting polymers with inorganic nanoparticles like oxides, possess outstanding properties due to synergistic effects of the component materials. This may include either new or improved electronic, mechanical, optical, thermal, magnetic or chemical properties. The properties of nanocomposites of such kind are strongly dependent on concentration of polymer. The state of dispersion of nanoparticles in the polymeric matrix has often a large impact on the properties of polymers [3]. The dramatically larger chain-particle interface area in the case of nanocomposites makes effects appearing negligible in microcomposites and very prominent in nanocomposites. The addition of nanoscale fillers could improve the electrical and dielectric properties of the host polymers[4]. Polypyrrole is one of the most attractive polymers due to its special transport properties, superior conductivity, electrochemical reversibility, facile synthesis, and good environmental stability [5]. Nano crystalline ferrites are materials of considerable interest due to their unique dielectric, magnetic, electrical and optical properties, which make them appealing both from scientific and technological point of view. We have selected Nickel zinc ferrites (Nickel zinc iron oxide) nanoparticles due to their importance in wide range of applications in lithium batteries, highdensity information storage devices, radar absorbing materials, magnetic fluids etc.[6]. This paper reports Synthesis, Characterization and DC conductivity of nanocomposites formed by blending Polypyrrole with $\mathrm{NiZnFe}_{2} \mathrm{O}_{4}$ nanoparticles. 


\section{Experimental Details}

Pyrrole, Ammonium Persulphate, Nickel Zinc iron oxide nanoparticles were purchased from Sigma Aldrich. The particle size of nickel zinc iron oxide nanoparticle is $<100 \mathrm{~nm}$. The pyrrole monomer was purified by distilling under reduced pressure and used. All chemicals were of analytical grade and used as received without any further treatment.

\subsection{Synthesis of Polypyrrole}

Polypyrrole (PPy) was synthesized by in-situ polymerization of monomer Pyrrole in the presence of oxidising agent ammonium persulphate. $0.3 \mathrm{M}$ Pyrrole taken in a round bottomed flask which was placed in an ice tray mounted on a magnetic stirrer. $0.6 \mathrm{M}$ Ammonium persulphate was added drop wise using a burette to the above $0.3 \mathrm{M}$ Pyrrole. The reaction was carried out for 5 hours under continuous stirring maintaining a temperature $0^{\circ} \mathrm{C}$ to $5^{\circ} \mathrm{C}$. The resulting precipitate was collected after filtration by the method of suction; the black Polypyrrole powder thus obtained was dried in a hot air oven and subsequently in a muffle furnace at a temperature of $100^{\circ} \mathrm{C}$. The final product was $\sim 2.15 \mathrm{~g}$, taken as $100 \mathrm{wt} . \%$ [7].

\subsection{Synthesis of Polypyrrole / $\mathrm{NiZnFe}_{4} \mathrm{O}_{4}$ Nanocomposites}

$\mathrm{PPy} / \mathrm{NiZnFe}_{2} \mathrm{O}_{4}$ nanocomposites were synthesized by the same method. For $0.3 \mathrm{M}$ Pyrrole solution, $0.215 \mathrm{~g}(10 \mathrm{Wt} \%)$ of $\mathrm{NiZnFe}_{2} \mathrm{O}_{4}$ was added and mixed thoroughly in a round bottomed flask. O.6M ammonium persulphate was added drop-wise with the help of burette to the above solution as described above and the entire procedure is repeated to get $10 \mathrm{wt} \%$ composite. Similarly $20 \mathrm{wt} . \%, 30 \mathrm{wt} . \%, 40 \mathrm{wt} . \%$ and $50 \mathrm{wt} . \%$ $\mathrm{PPy} / \mathrm{NiZnFe}_{2} \mathrm{O}_{4}$ nanocomposites are prepared by adding $0.430,0.600,0.860$, and $1.075 \mathrm{~g}$ of $\mathrm{NiZnFe}_{2} \mathrm{O}_{4}$ nanopowder to $0.3 \mathrm{M}$ Pyrrole solution and repeating all the steps as described above. The pure Polypyrrole and $\mathrm{PPy} / \mathrm{NiZnFe}_{2} \mathrm{O}_{4}$ nanocomposites are pressed in the form of pellets of thickness approx $2 \mathrm{~mm}$ and diameter $10 \mathrm{~mm}$ , using hydraulic press by applying 10-12 tons of pressure. The pellets of the synthesized composites are coated with silver paste on either side of the surfaces to obtain better contacts for conductivity measurements [7-8].

\subsection{Conductivity Measurements}

The DC conductivity was measured as a function of temperature by two Probe method using Keithley electrometer in the temperature range $303 \mathrm{~K}$ to $473 \mathrm{~K}$. A constant voltage was applied to the pellets of samples and the current through the sample was measured using digital nanoammmeter. The temperature of the sample was measured by a temperature control unit provided with Chromel Alumel thermocouple. The conductivity was determined using the relation $\sigma=\frac{t}{R A} \quad$ where ' $\mathrm{t}$ ' is the thickness of the sample,' $\mathrm{R}$ ' is the resistance and 'A' is area of cross section of the samples[9].

\section{Characterizations}

The composites thus obtained were characterized by X-ray diffraction (XRD), Fourier transform infrared spectrometer (FTIR) and scanning electron microscopic (SEM) techniques. The X-ray diffraction patterns of Polypyrrole and $\mathrm{PPy} / \mathrm{NiZnFe}_{2} \mathrm{O}_{4}$ were recorded on X-ray Diffractometer (Shimadzu 7000s) using $\mathrm{CuK}_{\alpha}$ radiation $(\lambda=1.5418 \AA)$ in the $2 \theta$ range $10^{\circ}-80^{\circ}$ Where ' $\theta$ ' is the angle of diffraction[10]. The FTIR spectra of PPy and PPy/ $\mathrm{NiZnFe}_{2} \mathrm{O}_{4}$ composites were recorded on FTIR (Perkin Elmer) spectrometer in $\mathrm{KBr}$ medium at room temperature. The SEM micrographs of the PPy and $\mathrm{PPy} / \mathrm{NiZnFe}_{2} \mathrm{O}_{4}$ nanocomposites are recorded using Scanning Electron Microscope (Vega 3 Tescan).

\subsection{XRD Analysis}

\section{Results And Discussions}

The XRD spectrum of pure PPy is represented in Fig.1a. It shows a broad peak at $2 \theta=26.88^{\circ}$ which is the characteristic peak of amorphous PPy.[11] This broadening of peaks can be ascribed to the scattering of Xrays from PPy chains at the interplanar spacing [12-13].

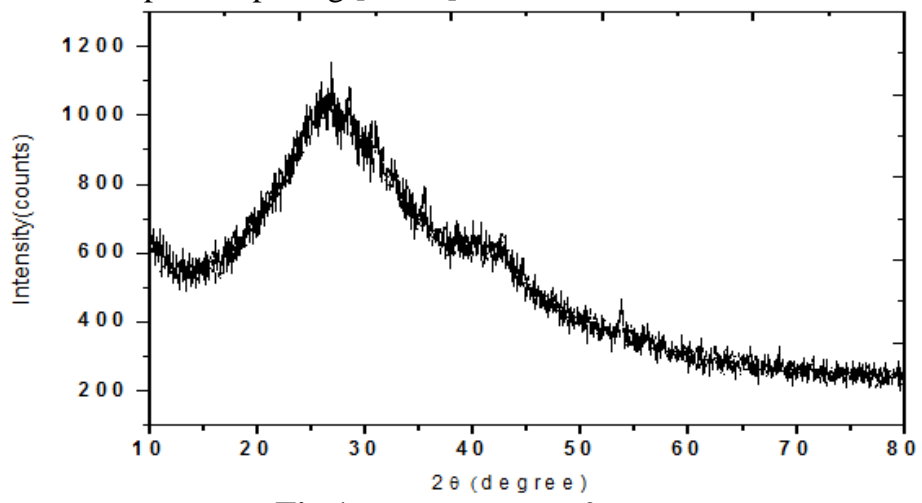

Fig.1a XRD pattern of PPy 
The observed XRD patterns of PPy/ $\mathrm{NiZnFe}_{2} \mathrm{O}_{4}$ nanocomposites and $\mathrm{NiZnFe}_{2} \mathrm{O}_{4}$ are as shown in Fig.1b and 1c respectively. The appearance of the sharp peaks in the composite may indicate some degree of crystallinity in the composite. The pattern shows crystalline spinel cubic structure and is in good agreement with JCPDS Card 08-0234[14]. On insertion of nanofiller $\mathrm{NiZnFe}_{2} \mathrm{O}_{4}$ to polymer PPy a number of new small peaks are observed at lower angle side, these small new peaks are attributed to the formation of polymer-nanoparticle interactions. The (hkl) values for some of the most prominent peaks are (311),(400),(511) and (440) which corresponds to the interplanar distance (d) $2.5330 \AA, 2.0971 \AA, 1.6151 \AA$ and $1.4814 \AA$ respectively. The lattice constant (a) has been calculated by using the relation $d=\frac{a}{\sqrt{h^{2}+k^{2}+l^{2}}}$ and found to be $8.401 \AA$, $8.388 \AA, 8.392 \AA$, and $8.380 \AA$ for the planes (311),(400),(511) and (440) respectively. This value agrees with the standard value $8.443 \AA$ [14]. The average crystallite size (D) as estimated from line broadening in X-ray diffraction using Scherrer's formula, $D=k \lambda / \beta \cos \theta$ was found to be $15 \mathrm{~nm}$ for nanocomposites. where ' $\mathrm{k}$ ' is the phase factor $=$ 0.9 , ' $\lambda$ ' is the wavelength of X-rays $=1.54 \AA, \quad$ ' $\beta$ ' is the Full width at half maximum (FWHM) and ' $\theta$ ' is the angle of diffraction.

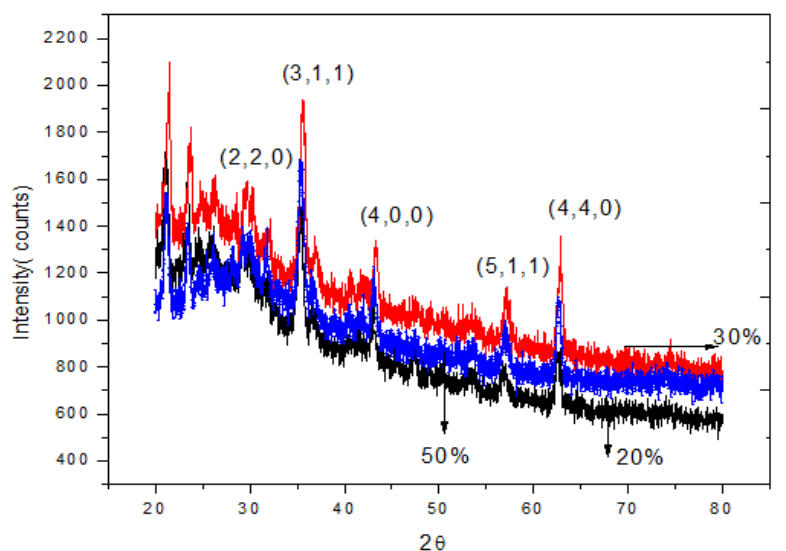

Fig.1b. XRD pattern of $\mathrm{PPy} / \mathrm{NiZnFe}_{2} \mathrm{O}_{4}$

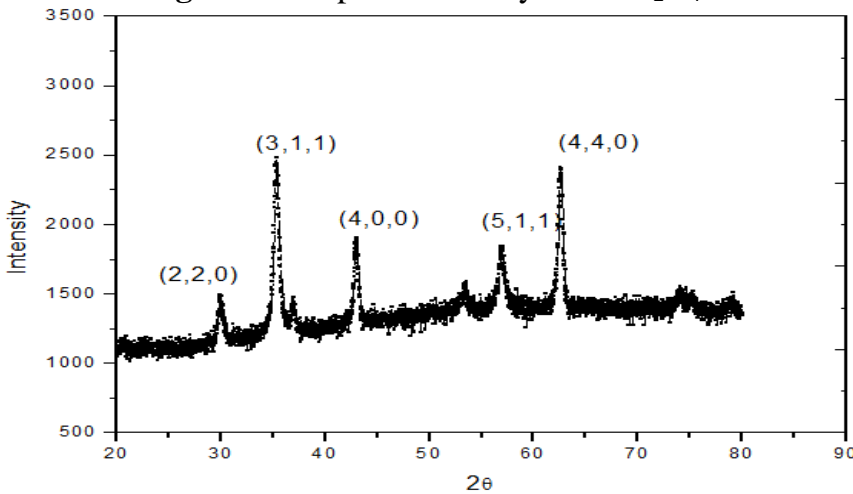

Fig.1c. XRD pattern of $\mathrm{NiZnFe}_{2} \mathrm{O}_{4}$

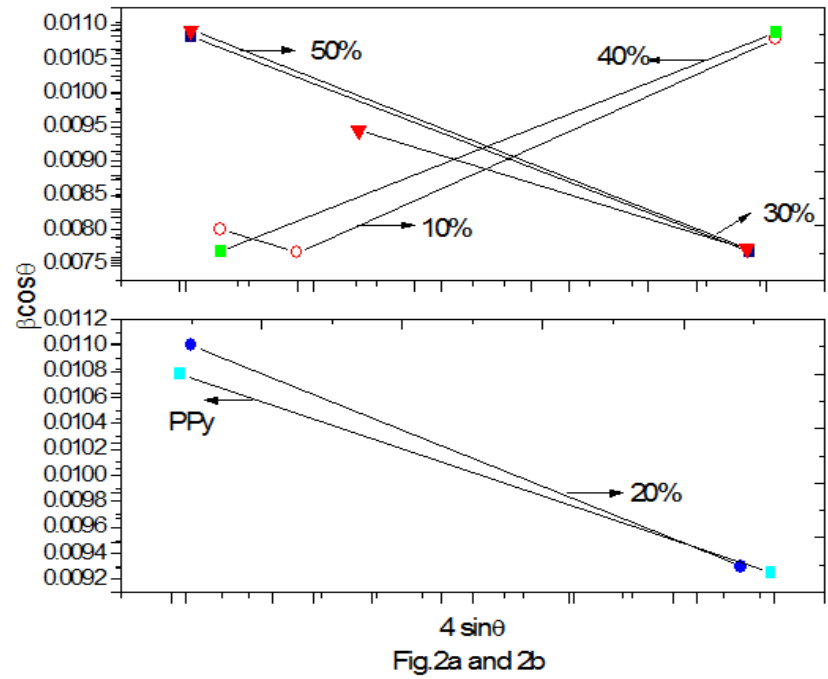

Fig. $2 \mathrm{a}$ and $2 \mathrm{~b}$ 
It is observed that the peaks are shifted towards lower diffraction angles. This is attributed to the lattice stretching which may be due to the linkage between host polymer PPy and nanofiller $\mathrm{NiZnFe}_{2} \mathrm{O}_{4}$ resulting in tensile stress and hence strain. The lattice strain was estimated using $\mathrm{W}-\mathrm{H}$ plots. The slope of the line gives strain and from the intercept $(\mathrm{k} \lambda / \mathrm{D})$ on $\mathrm{Y}$-axis crystallite size (D) is calculated. The crystallite size calculated from W-H plot was found to be in the range of $15 \mathrm{~nm}$ to $20 \mathrm{~nm}$ and agrees with Scherrer's equation. The lattice parameters evaluated from XRD are tabulated in Table 1.

Table 1. Crystal Parameters of $\mathrm{NiZnFe}_{2} \mathrm{O}_{4}$ evaluated by XRD

\begin{tabular}{|c|c|c|c|c|}
\hline SL.No. & $\begin{array}{l}\text { Size by } \\
\text { Scherrer } \\
(\mathrm{nm})\end{array}$ & $\begin{array}{l}\text { Size by W-H plot } \\
(\mathrm{nm})\end{array}$ & Strain & $\begin{array}{l}\text { Lattice } \\
\text { Constant(a) } \\
\AA\end{array}$ \\
\hline PPy/10\% & 15.96 & 48.80 & 0.00654 & 8.4327 \\
\hline PPy $/ 20 \%$ & 13.86 & 11.32 & -0.00135 & 8.3716 \\
\hline PPy/30\% & 14.68 & 13.75 & -0.00028 & 8.3576 \\
\hline PPy/40\% & 18.35 & 28.28 & 0.00164 & 8.3852 \\
\hline PPy $/ 50 \%$ & 14.65 & 09.88 & -0.00265 & 8.3902 \\
\hline PPy & 21.90 & 24.80 & -0.00031 & 8.2450 \\
\hline $\mathrm{NiZn} \mathrm{Fe}_{2} \mathrm{O}_{4}$ & 16.40 & 10.78 & -0.00244 & 8.4000 \\
\hline
\end{tabular}

\subsection{FTIR Analysis}

The FTIR spectra of Pure PPy, PPy / NiZnFe $\mathrm{O}_{4}$ and $\mathrm{NiZnFe}_{2} \mathrm{O}_{4}$ are shown in Fig.3. FTIR spectra of pure PPy shows main characteristic peak at $675 \mathrm{~cm}^{-1}$ which corresponds to ring deformation, peaks at $817 \mathrm{~cm}^{-1}$ and $917.75 \mathrm{~cm}^{-1}$ correspond to $\mathrm{C}-\mathrm{H}$ deformation, peak at $1074 \mathrm{~cm}^{-1}$ refers $\mathrm{C}-\mathrm{H}$ in plane bend and peak at 1198 $\mathrm{cm}^{-1}$ corresponds to ring breathing, the peak at $1365 \mathrm{~cm}^{-1}$ is due to fundamental vibrations of PPy ring, the peaks at $1547 \mathrm{~cm}^{-1}$ and $1489.4 \mathrm{~cm}^{-1}$ attributes to $\mathrm{C}=\mathrm{C}$ stretching, the occurrence of small peak at $3438 \mathrm{~cm}^{-1}$ is assigned to the presence of $\mathrm{N}-\mathrm{H}$ stretching frequencies. The peaks observed in the present work match well with the previous studies [15-16]. In addition to these peaks two peaks are noticed at $617 \mathrm{~cm}^{-1}$ and $1244 \mathrm{~cm}^{-1}$ which are assigned to $\mathrm{C}=\mathrm{O}$ out-of-plane bend and $\mathrm{C}-\mathrm{N}$ stretch respectively. The sharp peak appeared at $593.4 \mathrm{~cm}^{-1}$ in $\mathrm{NiZnFe}_{2} \mathrm{O}_{4}$ disappear in $\mathrm{PPy} / \mathrm{NiZnFe}_{2} \mathrm{O}_{4}$ nanocomposite which may be due to the dominating phase of PPy in the formed polymer nanocomposites [17]

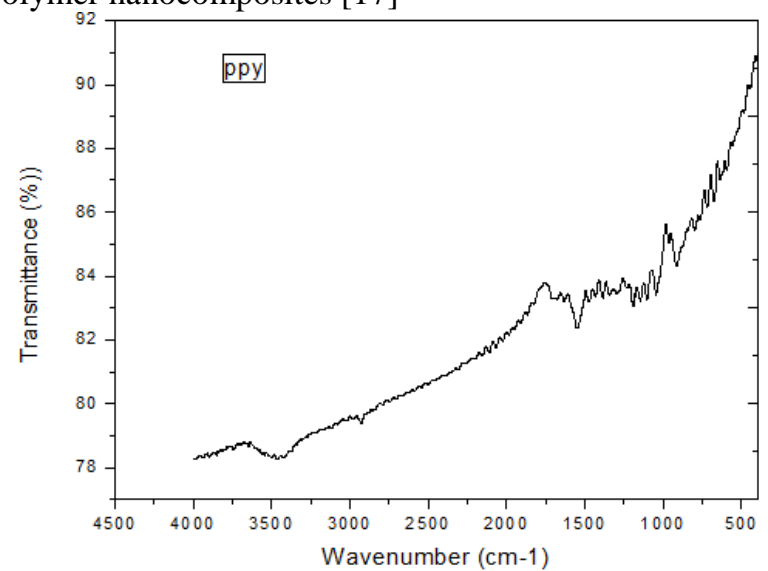

Fig.3 FTIR spectra of PPy

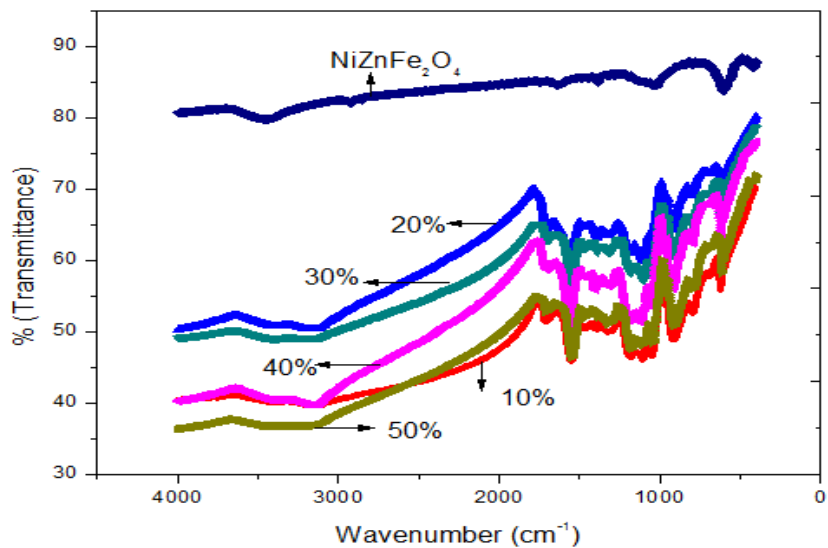

Fig.3 FTIR spectra of PPy/NiZnFe ${ }_{2} \mathrm{O}_{4}$ and $\mathrm{NiZnFe}_{2} \mathrm{O}_{4}$ nanocomposites 


\subsection{SEM Analysis}

The SEM micrographs of PPy, PPy/ $\mathrm{NiZnFe}_{2} \mathrm{O}_{4}-20 \%$, PPy/NiZnFe $\mathrm{O}_{4}-30 \%, \mathrm{PPy} / \mathrm{NiZnFe}_{2} \mathrm{O}_{4}-50 \%$ and $\mathrm{NiZnFe}_{2} \mathrm{O}_{4}$ are shown in Fig.3a, 3b, 3c, 3d and 3e respectively. It is clear from the SEM micrographs of pure PPy that, it has clusters of spherical shaped particles. Morphologically PPy shows the existence of small globules and porous which can be beneficial for diffusion of ions. Comparison of Fig.4b, 4c, 4d and 4e with Fig.4a, shows the confirmation of the distribution of $\mathrm{NiZnFe}_{2} \mathrm{O}_{4}$ in the polymer chain. At composition of $50 \mathrm{wt} \%$ a complete chain pattern is observed, with the chains connecting the nanosized oxide particles, enabling better conductivity

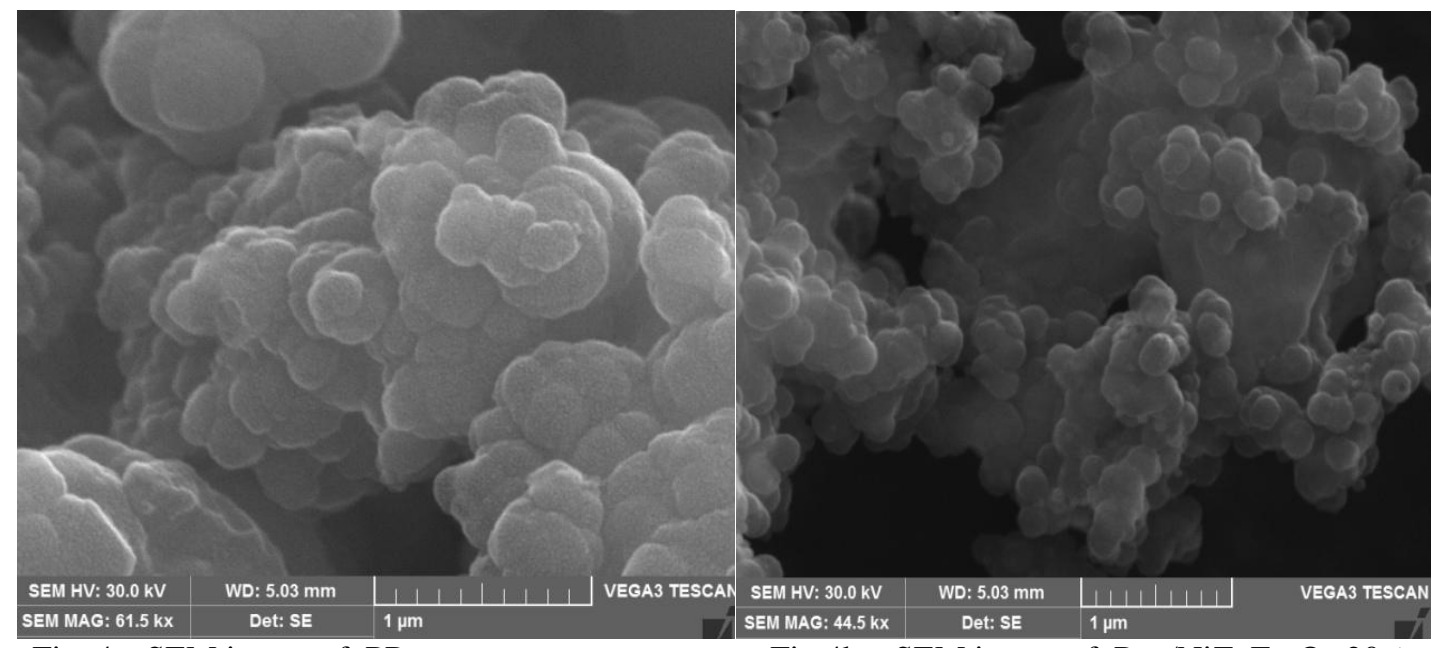

Fig. 4a. SEM image of PPy

Fig.4b. . SEM image of Ppy/NiZnFe ${ }_{2} \mathrm{O}_{4}-20 \%$
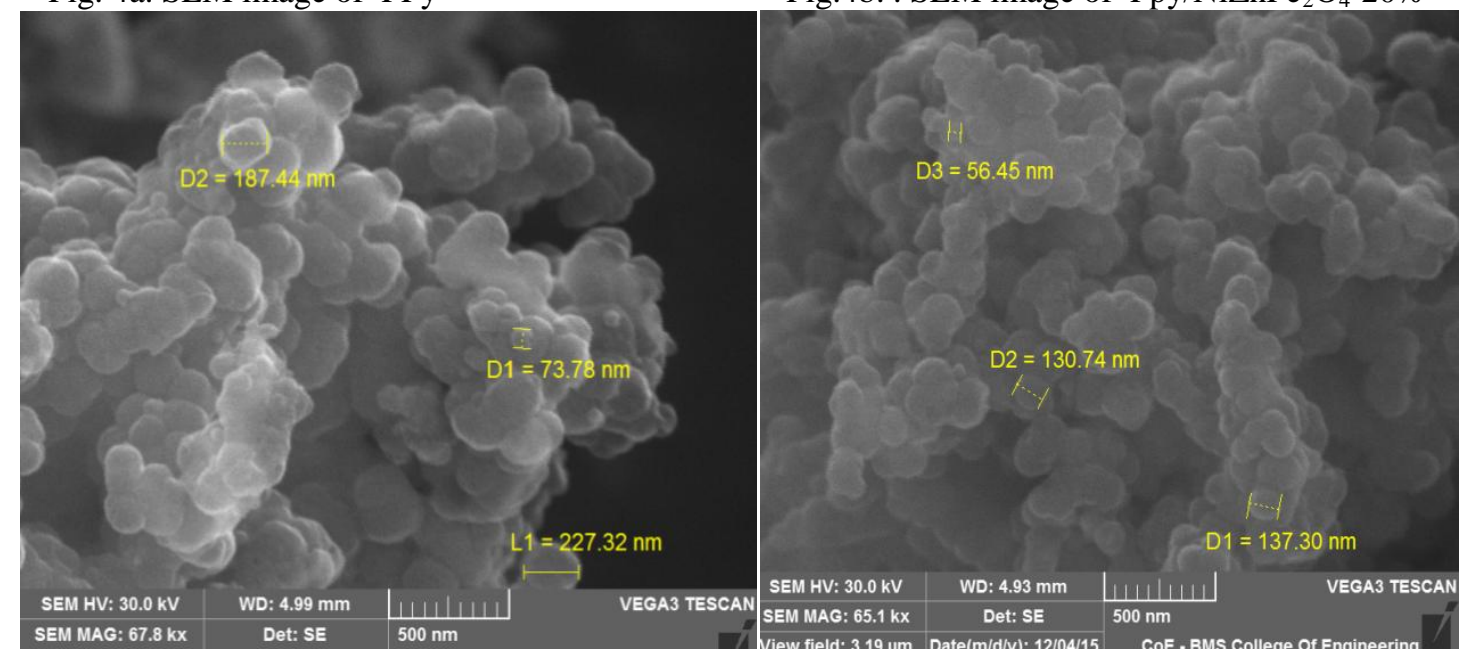

Fig.4c.SEM image of Ppy/NiZnFe ${ }_{2} \mathrm{O}_{4}-30 \%$

Fig.4d. SEM image of Ppy/NiZnFe $2 \mathrm{O}_{4}-40 \%$

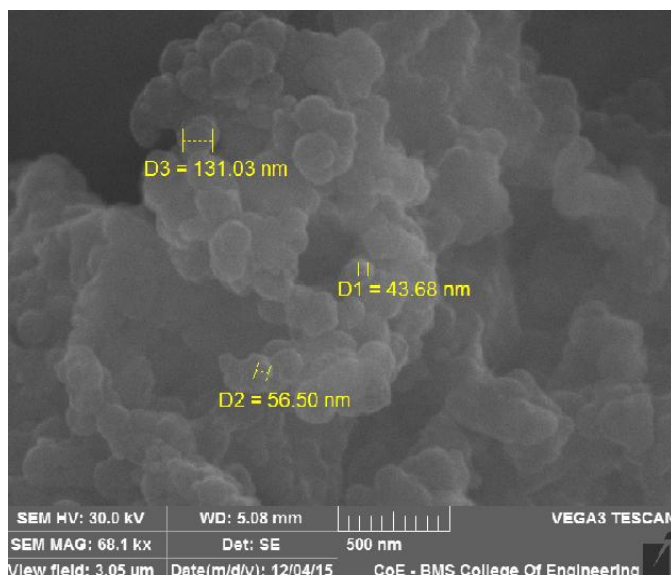

Fig.4e. SEM image of Ppy/NiZnFe $\mathrm{O}_{4}-50 \%$

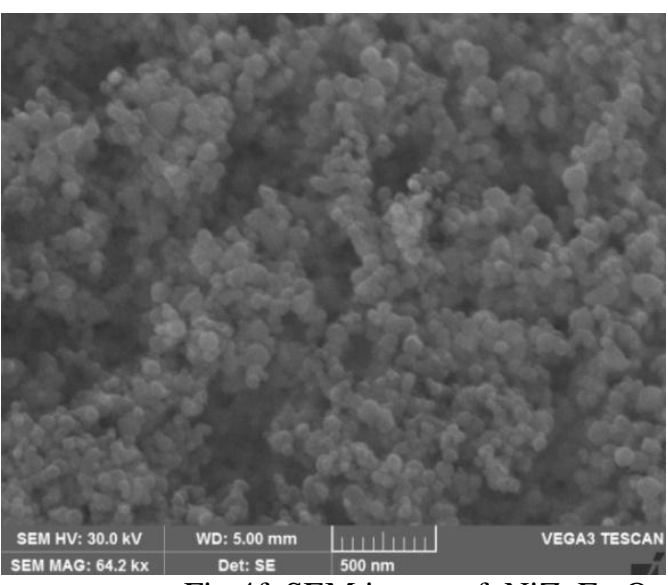

Fig.4f. SEM image of $\mathrm{NiZnFe}_{2} \mathrm{O}_{4}$ 


\subsection{DC conductivity}

DC electrical conductivity is one of the useful characterization techniques to understand conductivity mechanism. The variation of DC conductivity of nanocomposites with temperature was studied and reported. It was observed that the DC conductivity of the nanocomposites of all compositions except 50\% are not much affected with the inclusion of nanoparticles, but the DC conductivity of $\mathrm{PPy} / \mathrm{NiZnFe}_{2} \mathrm{O}_{4}-50 \%$ increased remarkably by almost $200 \%$ when compared to base pure PPy, The objective of the present study is to evaluate the activation energy of the derived PPy nanocomposites by measuring DC conductivity of individual composite material and examine the extent of increase of activation energy, which is regarded as a proof of composite formation with enhanced electrical conductivity characteristics.

\subsection{Activation Energy}

The variation of conductivity (' $\sigma$ ') with temperature is explained by Arrhenius equation given by $\sigma=$ $A \exp \left(\frac{-E_{a}}{k T}\right)$

Where ' $\mathrm{A}$ ' is the pre exponential factor, ' $E_{a}$ ' is the activation energy, ' $k$ ' is the Boltzmann constant and ' $\mathrm{T}$ ' is the temperature in $\mathrm{K}$.

The activation energy is estimated from the slope of Arrhenius plot of $(\ln \sigma)$ against (1/T) [18-19].

Activation energy $\left(\mathrm{E}_{\mathrm{a}}\right)=-$ slope $\mathrm{x} k$ (Slope being negative, activation energy is positive).

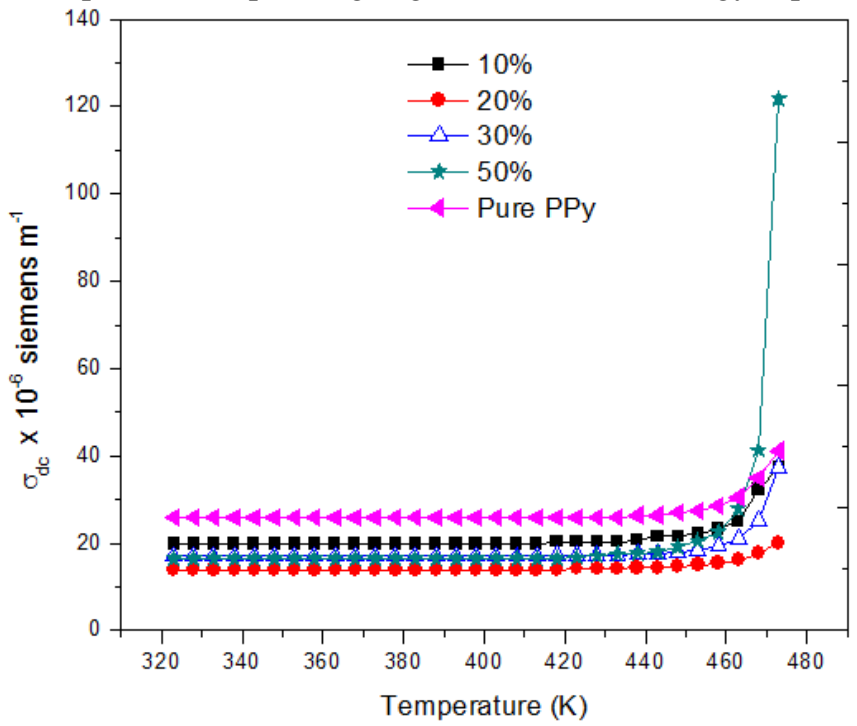

Fig.6a Variation of Conductivity with temperature for $\mathrm{Ppy} / \mathrm{NiZnFe}_{2} \mathrm{O}_{4}$ nanocomposites

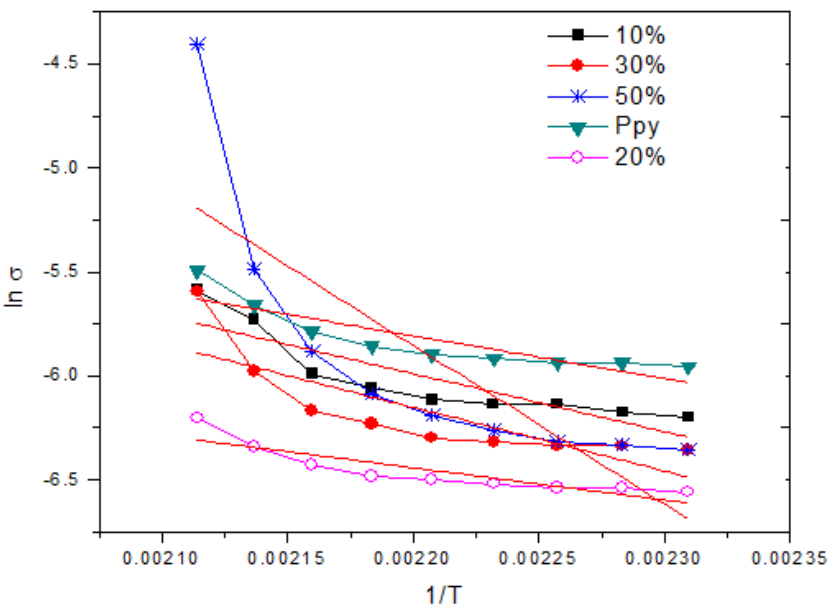

Fig.6b Arrhenius plots for $\mathrm{Ppy} / \mathrm{NiZnFe}_{2} \mathrm{O}_{4}$ nanocomposites

Fig. $6 \mathrm{a}$ and $6 \mathrm{~b}$ represents the variation of DC conductivity with temperature and activation energy plots for $10 \mathrm{wt} . \%, 20 \mathrm{wt} \%, 30 \mathrm{wt} . \%, 50 \mathrm{wt} . \%$ and pure PPy respectively It has been observed that the $\sigma_{\mathrm{dc}}$ curves are linear from room temperature until a temperature $433 \mathrm{~K}$ and then increases exponentially indicating semiconducting type of behaviour. So the activation energy is calculated from the slopes of Arrhenius plots (Fig. 6b) for temperature range $433 \mathrm{~K}-473 \mathrm{~K}$. The activation energy ' $\mathrm{E}_{\mathrm{a}}$ ' was found to be $0.1769 \mathrm{eV}$ for pure PPy. 


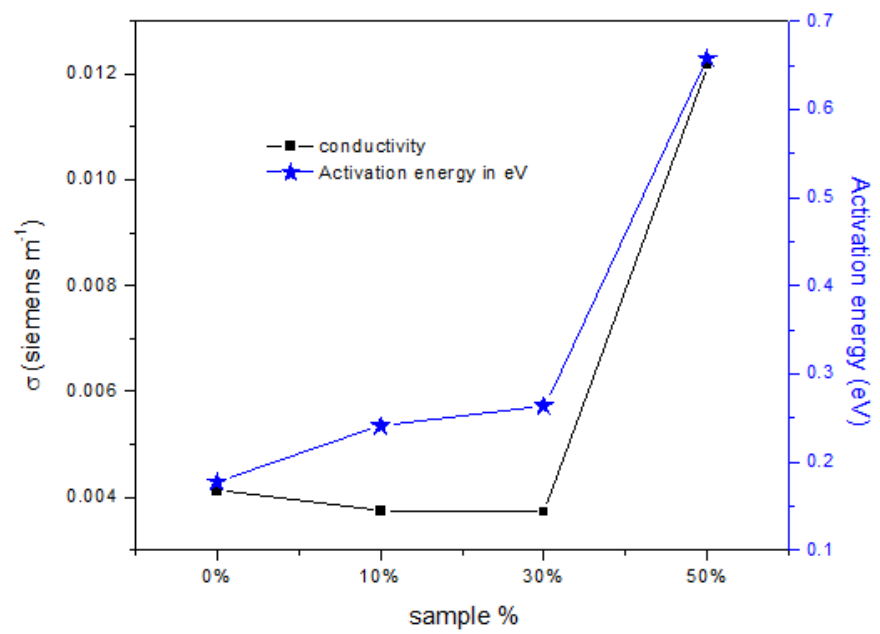

Fig.7 Variation of Conductivity and Activation energy with sample \% for Ppy/NiZnFe ${ }_{2} \mathrm{O}_{4}$ nanocomposites

On addition of nanofiller into the polymer matrix, It is noticed that the nanocomposites of different compositions behaved differently. The activation energy ' $\mathrm{Ea}$ ' for nanocomposites of $10 \mathrm{wt} . \%,, 30 \mathrm{wt} . \%$, and $50 \mathrm{wt} \%$ was found to be $0.2408,0.2632 \mathrm{eV}$ and $0.6580 \mathrm{eV}$ respectively. It is seen that $\mathrm{PPy} / \mathrm{NiZnFe}_{2} \mathrm{O}_{4}-50 \mathrm{wt}$. \% composites of activation energy $0.6580 \mathrm{eV}$ have highest conductivity of $121.8 \times 10^{-6}$ Siemens $\mathrm{cm}^{-1}$. Fig.8 represents the variation of ' $\sigma$ ' at $473 \mathrm{~K}$ and ' $\mathrm{E}_{\mathrm{a}}$ ' for different wt $\%$ composites.

From the conductivity graphs(fig.6a) it is clear that the conductivity is not same for all composites even at room temperature, Though conductivity increases with increase in activation energy, the room temperature conductivity values indicate that the conductivity at room temperature is controlled by the topology of the system, (i.e., by the connectivity of the conductive network in the composites). This confirms that the DC conductivity is not only a function of temperature as exhibited by Arrhenius equation but also depends on the dielectric constant at room temperature and the conductivity is not a measure of activation energy alone[20].

The Highest conductivity (at 473K), the conductivity at room temperature and activation energy for pure PPy and nanocomposites of different wt $\%$ are compared in Table. 2 Since the conductivity $(\ln \sigma)$ is increased in the case of nanocomposites, the nanostructured nickel Zinc iron oxide (50wt.\%) filler material distributed in the filler matrix is creating clear conducting path for the flow of current thereby increasing the conductivity of the composite material.

Table.2 DC Conductivity and Activation energy, of Nanocomposites at 303K, and at 473K

\begin{tabular}{|c|c|c|c|c|}
\hline $\begin{array}{c}\text { SL. } \\
\text { No }\end{array}$ & Sample & $\begin{array}{c}\text { Conductivity at } 303 \mathrm{~K} \sigma_{0} \\
\left.(\text { Siemens cm })^{-1}\right)\end{array}$ & $\begin{array}{l}\text { Max. Conductivity at 473K } \sigma_{\mathrm{dc}} \\
\left(\text { Siemens cm }{ }^{-1}\right)\end{array}$ & $\begin{array}{c}\text { Activation } \\
\text { energy } \\
\mathrm{E}_{\mathrm{a}} \text { in eV }\end{array}$ \\
\hline 1 & $\begin{array}{c}\text { Pure PPy } \\
0 \%\end{array}$ & $26.40 \times 10^{-6}$ & $41.20 \times 10^{-6}$ & 0.1769 \\
\hline 2 & $10 \%$ & $19.99 \times 10^{-6}$ & $37.38 \times 10^{-6}$ & 0.2408 \\
\hline 3 & $20 \%$ & $13.87 \times 10^{-6}$ & $20.23 \times 10^{-6}$ & 0.1151 \\
\hline 4 & $30 \%$ & $17.01 \times 10^{-6}$ & $37.16 \times 10^{-6}$ & 0.2632 \\
\hline 5 & $40 \%$ & $14.42 \times 10^{-6}$ & $28.07 \times 10^{-6}$ & 0.1772 \\
\hline 6 & $50 \%$ & $16.41 \times 10^{-6}$ & $121.75 \times 10^{-6}$ & 0.6580 \\
\hline
\end{tabular}

\section{Conclusions}

This work highlights the processing of nanostructured $\mathrm{PPy} / \mathrm{NiZnFe}_{2} \mathrm{O}_{4}$ nanocomposites by in-situ polymerization and feasibility of using such composite materials as a better electrical conducting material. The formation of composites was confirmed by XRD, FTIR and SEM techniques. The crystallite size calculated from XRD studies was found to be in the range of $15 \mathrm{~nm}$ to $20 \mathrm{~nm}$. The lattice strain was measured and found to be in the range -0.00028 to 0.0064 . The conductivity of the polymer nanocomposites show temperature dependence where it increases with the increasing temperature and shows the Arrhenius behaviour. Activation energy is measured by Arrhenius plots. Experimental results indicate that a significant increase in DC conductivity and activation energy is achieved for particular nanocomposite (50wt.\%) when compared to their counter base polymers. Such polymer nanocomposite materials may find applications in sensors, electronic devices, electromagnetic shielding, microwave absorbers etc. 


\section{Acknowledgements}

The authors would like to acknowledge Dr. Mallikarjuna Babu, Principal, B.M.S. College of Engineering, Bangalore and Dr. Pranesh, HOD, Dept. of Physics, B.M.S. College of Engineering, Bangalore for their support in carrying out this work.

\section{References}

[1] DR Paul, LM Robeson (2008) Polymer nanotechnology: Nanocomposites Polymer 49: 3187-3204

[2] H Arami, M Mazloumi, R Khalifehzadeh, S Hojjati Emami, SK Sadrnezhaad (2007) Polypyrrole/multiwall carbon nanotube nanocomposites electro polymerized on copper substrate Materials Letters. 61: 4412-4415

[3] L Mikoliunaite, et.al ; (2014) Development of gold nanoparticle-polypyrrole nanocomposites chemija. 25:63-69

[4] J Jancar , (2010) Current Issues in Research on Structure-Property Relationships in Polymer Nanocomposites Polymer. 51: 33213343.

[5] Lin-Xia Wang, Xin-Gui Li , Yu-Liang Yang, (2001) Preparation, properties and applications of polypyrroles Reactive \& Functional Polymers 47:125-139

[6] G S Shahane, Ashok Kumar, R.P. Pant, and Krishnan Lal,(2009) Structural and Magnetic Properties Of Ni-Zn Ferrite Nanoparticles International Conference on Advanced Nanomaterials and Nanotechnology (ICANN-2009) 1276: 374 - 380

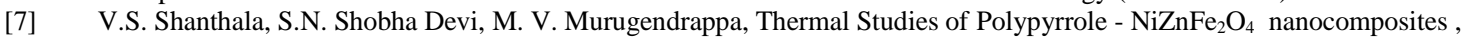
International journal of Innovative Research in Technology, (2016) 3 Issue 1: 198-203

[8] M.V. Murugendrappa and Ambika Prasad, (2007) Chemical synthesis, characterization and dc conductivity studies of polypyrrole Fe2O3 composites J of Applied Polymer Science. 103:2797-2801

[9] K. Rama Krishna, K. Vijaya Kumar, D Ravinder, (2012) Structural and Electrical Conductivity studies in Nickel zinc Ferrite, Advances in Materials Physics and Chemistry $2: 185-191$.

[10] Sangappa K Ganiger, Chaluvaraju B V and Murugendrappa M V (2014) . Synthesis, Characterization and Dielectric Study of Polypyrrole/Sodium Metavanadate (Ceramic) Composites International Journal of Engineering Sciences \& Research Technology 3: 130-136

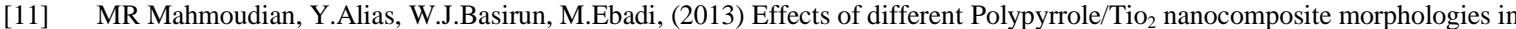
polyvinyl butyral coatings for preventing the corrosion of mile steel, Applied Surface Science. 268:302-311.

[12] M A. Chougule et.al; (2011) Synthesis and Characterization of Polypyrrole (ppy) thin Films, Soft Nanoscience Letters. 1: 6-10.

[13] VT Bhugul, G.N. Choudhari, (2015). Synthesis and Studies on Nanocomposites of polypyrrole Al-doped zinc oxide nanoparticles International Journal of Scientific and Research Publications. $5: 1-5$

[14] AU Chaudhry, R Bhola, V Mittal, B Mishra, (2014) $\mathrm{Ni}_{0.5} \mathrm{Zn}_{05} \mathrm{Fe}_{2} \mathrm{O}_{4}$ as a Potential corrosion Inhibitor for API 5L X80 Steel in Acidic Environment, Int. J. Electrochem. Sci $9: 4478$ - 4492

[15] H K Chitte1, Narendra V. Bhat, Ajit V. Gore, Ganesh N. Shind. (2011) Synthesis of polypyrrole using Ammonium peroxy disulfate(APS) as oxidant together with some dopants for use in Gas sensors, Materials Sciences and Applications Materials Sciences and Applications. 2: 1491-1498.

[16] T K Vishnuvardhan, VR Kulkarni, C Basavaraja and S C Raghavendra, (2006) Synthesis, characterization and a.c. conductivity of polypyrrole/Y2O3 composites Bull Mater. Sci., $29: 77-83$

[17] Suyinh Wei et.al; (2011) Polypyrrole-Titania Nanocomposites Derived from Different Oxidants, Journal of Electrochemical Society. 158 : K205-K212

[18] Sudha L.K, Sukumar Roy, and K. Uma Rao, (2014) Evaluation of Activation Energy (Ea) Profiles of Nanostructured Alumina Polycarbonate Composite Insulation Materials International Journal of Materials, Mechanics and Manufacturing. 2 : 96-100.

[19] V Shaktawat, K.Sharma, N.S.Saxena, (2010). Structural and electrical characterization of protonic acid doped polypyrrole, Journal of Ovonic Research . 6: $239-245$.

[20] Shujahadeen B. Aziz and Zul Hazrin Z. Abidin, (2013) Electrical Conduction Mechanism in Solid Polymer Electrolytes: New Concepts to Arrhenius Equation Journal of Soft Matter. $2013: 1-8$

IOSR Journal of Applied Physics (IOSR-JAP) is UGC approved Journal with S1. No. 5010, Journal no. 49054.

V.S. Shanthala. "Characterization and Evaluation of Activation Energy for Dc Conductivity of Polypyrrole/Nickel Zinc Iron Oxide Nanocomposites." IOSR Journal of Applied Physics (IOSR-JAP) 9.4 (2017): 29-36. 